\title{
On isomorphism classes of a subclass of filiform Leibniz algebras in dimension 10
}

\begin{abstract}
Some classes of filiform Leibniz algebras arising from the naturally graded non Lie filiform Leibniz algebras were introduced by Ayupov and Omirov in 2006. One of them is the first class of filiform Leibniz algebras and has been denoted by FLBn in fixed dimension n.Rakhimov and Bekbaev have suggested an approach of classifying FLBn based on algebraic invariants method. In terms of classification, it has been classified up to dimension 9 and the main purpose of this paper is to use method of invariants and apply to dimension 10. To complete this goal, we set up an isomorphism criterion, here we introduced some new functions to avoid big expressions involved in isomorphism criterion. From the criterion some lists of disjoint subsets from FLB10 are obtained. These disjoint subsets are represented as a union of parametric family of orbits or just single orbits. For parametric cases, the invariants will be given.
\end{abstract}

Keyword: Filiform Leibniz algebras; Invariants; Isomorphism classes 